\title{
Coverage and Awareness of and Compliance with Mass Drug Administration for Elimination of Lymphatic Filariasis in Burdwan District, West Bengal, India
}

\author{
Rabindra Nath Roy, Aditya Prasad Sarkar, Raghunath Misra, Amitava Chakroborty, \\ Tusar Kanti Mondal, Kanad Bag \\ Community Medicine, Burdwan Medical College, India
}

\begin{abstract}
India adopted WHO's strategy of repeated rounds of mass drug administration (MDA) with diethylcarbamazine to eliminate lymphatic filariasis. The present study attempted to assess the coverage and awareness of and compliance with MDA for elimination of lymphatic filariasis in Burdwan district of India, following MDA round in July 2010. A cross-sectional study was conducted among the four randomly-selected clusters in the district of Burdwan, West Bengal, India, covering 603 individuals from 154 households, using a predesigned pretested schedule. The drug distribution coverage, compliance, and effective coverage were $48.76 \%, 70.07 \%$, and $34.16 \%$ respectively. Only $41.4 \%$ of the study population was aware of the MDA activity. This evaluation study noted that MDA is restricted to tablet distribution only. There is an urgent need to improve compliance with drug intake through strengthening of the awareness programme involving both government health workers and community volunteers.
\end{abstract}

Key words: Compliance; Coverage; Lymphatic filariasis; Mass drug administration; India

\section{INTRODUCTION}

Lymphatic filariasis (LF) is one of the most debilitating and disfiguring scourges among all diseases. Globally, 1.3 billion people are estimated to be at risk of infection, and some 120 million people are infected in 83 countries. The South-East Asia Region (SEAR) accounts for about $65 \%$ of the global population at risk and $50 \%$ of the infected people. Nine of the 11 countries in the region are known to be endemic for filariasis (1).

Filariasis has been a major public-health problem in India. The disease is reported to be endemic in 250 districts in 20 states and union territories (UTs) of India. About 600 million people are at risk of lymphatic filariasis in these districts. Indigenous cases of lymphatic filariasis have been reported from most of the states, including West Bengal. However, some north-western states/UTs, namely Jammu

Correspondence and reprint requests:

Dr. Rabindra Nath Roy

Community Medicine

Burdwan Medical College

India

Email: rabinroynew@gmail.com and Kashmir, Himachal Pradesh, Punjab, Haryana, Chandigarh, Rajasthan, Delhi, Uttaranchal and north-eastern states, namely Sikkim, Arunachal Pradesh, Nagaland, Meghalaya, Mizoram, Manipur, and Tripura, are stated to be free from indigenouslyacquired filarial infection (2).

The National Health Policy (2002) has envisaged elimination of lymphatic filariasis in India by 2015 (3). Elimination of LF means cessation of LF as a public-health problem, when the microfilaria (Mf) rate is less than one percent among the population in all areas of an endemic country and the children born after elimination of LF are free from circulating antigenaemia. Absence of antigenaemia among children is considered an evidence of the absence of transmission and new infection $(4,5)$. The strategy for achieving the goal of LF elimination is by annual mass drug administration (MDA) with diethylcarbamazine (DEC) to the entire population at risk and morbidity management of lymphedema, along with the other vector management strategies (2). MDA, in combination with other techniques, has already eliminated filariasis from Japan and South Korea and markedly reduced the trans- 
mission in China (3). The Government of India launched administration of annual single-dose DEC in 2004 and proposed the day of administration of MDA to be observed as National Filaria Day (NFD) every year in the endemic districts. Under this programme, a single-dose antifilarial drug DEC is administered in the dose of $6 \mathrm{mg} / \mathrm{kg}$ of body-weight to inhabitants in filaria-endemic areas, excluding children below 2 years of age, pregnant women, and seriously-ill persons. A sustainable high coverage ( $>85 \%$ ) in endemic areas for 5 years or more is required to achieve the interruption of transmission and elimination of the disease (2). Rate of coverage and consumption compliance are the most crucial factors in the success of MDA strategy (4).

Adverse drug reactions may decrease the compliance with drug consumption at the community level. It is of great advantage that the side-effects following DEC administration are mild or absent when the drug is given in daily doses of $6 \mathrm{mg} / \mathrm{kg}$ or less. The DEC consumption may be associated with minor side-effects in $1-10 \%$ of the treated persons, particularly among the carriers of microfilariae. The community is made aware of the temporary side-effects that may occur in the population who may be carriers of microfilariae. The non-specific drug-related reactions include: headache, anorexia, nausea, abdominal pain, vomiting, dizziness, weakness, or lethargy. These symptoms begin within 1-2 hour(s) of taking the drug and persist for a few hours. Specific parasite-related allergic reactions due to destruction of microfilariae and adult worms include: fever, local inflammations around dead worms, and pruritus. Most of these side-effects are self-limiting (4).

Different field studies indicated suboptimal performance in the coverage of annual DEC consumption by eligible population (6). Health education is instrumental for the awareness-generation and active participation of the community and forms an integral part of the elimination strategy. The knowledge gap with regard to the disease and prevailing attitudes toward the programme may be the causes of poor compliance with the consumption of drugs. WHO has recommended periodic evaluation of the status of implementation of MDA activities by independent experts.

With this background, the present study attempted to assess the coverage and community awareness of and compliance with MDA and some aspects of implementation of the programme.

\section{MATERIALS AND METHODS}

The study was conducted in Burdwan district of West Bengal, India where the MDA has been undertaken since 2005. Burdwan is a district situated in the central part of South Bengal having an area of 17,024 sq. $\mathrm{km}$, with average annual rainfall of $1,460 \mathrm{~mm}$ and temperature varying from $5{ }^{\circ} \mathrm{C}$ to $36{ }^{\circ} \mathrm{C}$. According to 2001 Census in India, the district had a population of $6,895,514$, of whom $48 \%$ were female. A cross-sectional descriptive study was conducted in July 2010. As per the guideline suggested by task force of the Government of India for evaluation of filaria elimination programme, four clusters were identified (three from rural and one from urban areas) for the study (7). The purpose of the population-based survey was to provide a coverage estimate that is statistically likely to be representative of the sampled population. The sampling design adopted in the study would provide an estimate of actual coverage to the accuracy of plus or minus $6.5 \%(8)$.

The Primary Health Centre (PHC) constituted the sampling frame at the first stage of sampling, and the villages under the PHC constituted the sampling frame at the second stage. As this was a postMDA sample survey for assessing drug coverage, care was taken to ensure representative response from individuals living in the clusters with variable reported coverage immediately preceding the MDA round. All PHCs in a district were classified into three strata according to reported coverage of MDA activities undertaken during 2010 (PHC area with $<50 \%$, between $50 \%$ and $80 \%$, and $>80 \%$ coverage). Total number of PHCs in the district was 106 in 2010. A complete list of the villages was prepared for each of the strata, and one village was selected randomly, using random number table from each stratum. As none of the PHCs reported coverage less than 50\% in the previous round, we selected two villages randomly from the stratum with reported $50-80 \%$ coverage. For urban area, one of the municipalities was selected randomly from the list of municipalities in the district and, subsequently, one ward was selected randomly from the selected municipality.

For the purpose of the survey, a central point was identified in each of these clusters, and the first house was selected randomly (any number between 1 and 9). The next house was selected having the nearest entrance. Thereafter, a minimum of 30 households were selected consecutively in this manner, covering a minimum of 150 persons from 
each of the clusters. Finally, 603 individuals from 154 households constituted the study population.

The head of the family or other responsible member present at the time of survey was interviewed with the help of predesigned, pretested semi-structured questionnaire. We collected data on drug distribution, consumption, side-effects following DEC consumption, and awareness of lymphatic filariasis, including MDA programme. Sources of information on MDA programme was assessed among those who knew about MDA programme.

All data were compiled and analyzed applying appropriate statistical method. The assessment was completed within three weeks of completion of the MDA round. Nine drug distributors and other healthcare providers associated with this programme were interviewed to generate data regarding programme implementation.

\section{Ethical clearance}

Ethical clearance was obtained from institutional ethical committee of Burdwan Medical College. The study was carried out on request of the Department of Health and Family Welfare, Government of West Bengal. According to the guideline issued by the Department, informed consent was obtained from the study population. The purpose of the research was communicated to the selected household members, and their oral informed consent was obtained before administering the questionnaire. They were assured of confidentiality and anonymity.

\section{Analysis}

The assessment in this study was made in terms of proportion of people who have actually received DEC tablets (i.e. coverage of drug distribution), those who have ingested the tablets (i.e. consumption of DEC among sampled population), compli- ance (proportion ingested at sufficient dose of DEC by those who received the tablets) and effective coverage (i.e. proportion ingested at appropriate dose of DEC by eligible individuals) in the selected areas. Coverage and consumption of DEC was further analyzed by age and sex. We entered data on Excel sheet and analyzed with MS Excel software.

\section{RESULTS}

There were 639 individuals in 154 households, of whom only 603 were eligible for administration of MDA at the time of drug distribution. These 603 individuals who were eligible for MDA constituted the study population. A little more than half $(52.57 \%)$ of the population eligible for MDA was female, and the majority (66.99\%) of population was in the age-group of 15 years and above. Children below two years, pregnant women, and severely-ill persons were excluded. Of 154 families, 134 (87\%) received drugs; rest of the families did not receive antifilarial drugs (Table 1 ).

\section{Coverage by age}

We analyzed the coverage of drug distribution, proportion of non-compliance, and effective coverage among different age-groups (Figure). It was observed that some people received the drugs but did not consume or consumed at suboptimal dose (non-compliance). The proportion of noncompliance varied between $14.59 \%$ and $15.69 \%$ among different age-groups. The proportion of effective coverage was $41.18 \%$ and $20 \%$ in $6-14$ years and 2-5 years age-group respectively.

\section{Reasons for non-consumption of drug}

Overall, $51.24 \%$ of eligible beneficiaries did not receive DEC tablets, and even some of those who received the drugs did not consume DEC in adequate dose (Table 2). No definite reason was stated as a cause of non-consumption in one-quarter of

\begin{tabular}{|c|c|c|c|c|c|c|}
\hline Sex & $\begin{array}{c}\text { Eligible } \\
\text { population }\end{array}$ & $\begin{array}{c}\text { No. of eligible } \\
\text { people given DEC }\end{array}$ & $\begin{array}{l}\text { Drug distribution } \\
\text { coverage (\%) }\end{array}$ & $\begin{array}{l}\text { No. of people } \\
\text { consuming } \\
\text { DEC }\end{array}$ & Compliance (\%)* & $\begin{array}{c}\text { Effective } \\
\text { coverage (\%) }\end{array}$ \\
\hline Male & 286 & 141 & 49.30 & 85 & 60.28 & 29.72 \\
\hline Female & 317 & 153 & 48.26 & 121 & 79.08 & 38.17 \\
\hline Total & 603 & 294 & 48.76 & 206 & 70.07 & 34.16 \\
\hline \multicolumn{7}{|c|}{$\begin{array}{l}\chi^{2}=0.03 \text { (Drug distribution coverage among males and females), } \mathrm{df}=1, \mathrm{p}=0.86 ; \chi^{2}=11.49 \text { (Compliance } \\
\text { among males and females), } \mathrm{df}=1, \mathrm{p}=0.00 ;{ }^{*} \text { Compliance in percentage }=\text { Number of people who had ingest- } \\
\text { ed sufficient dose of DEC tablets/Total no. of people who had received the DEC tablets } \times 100 ; \mathrm{df}=\text { Degree } \\
\text { of freedom }\end{array}$} \\
\hline
\end{tabular}




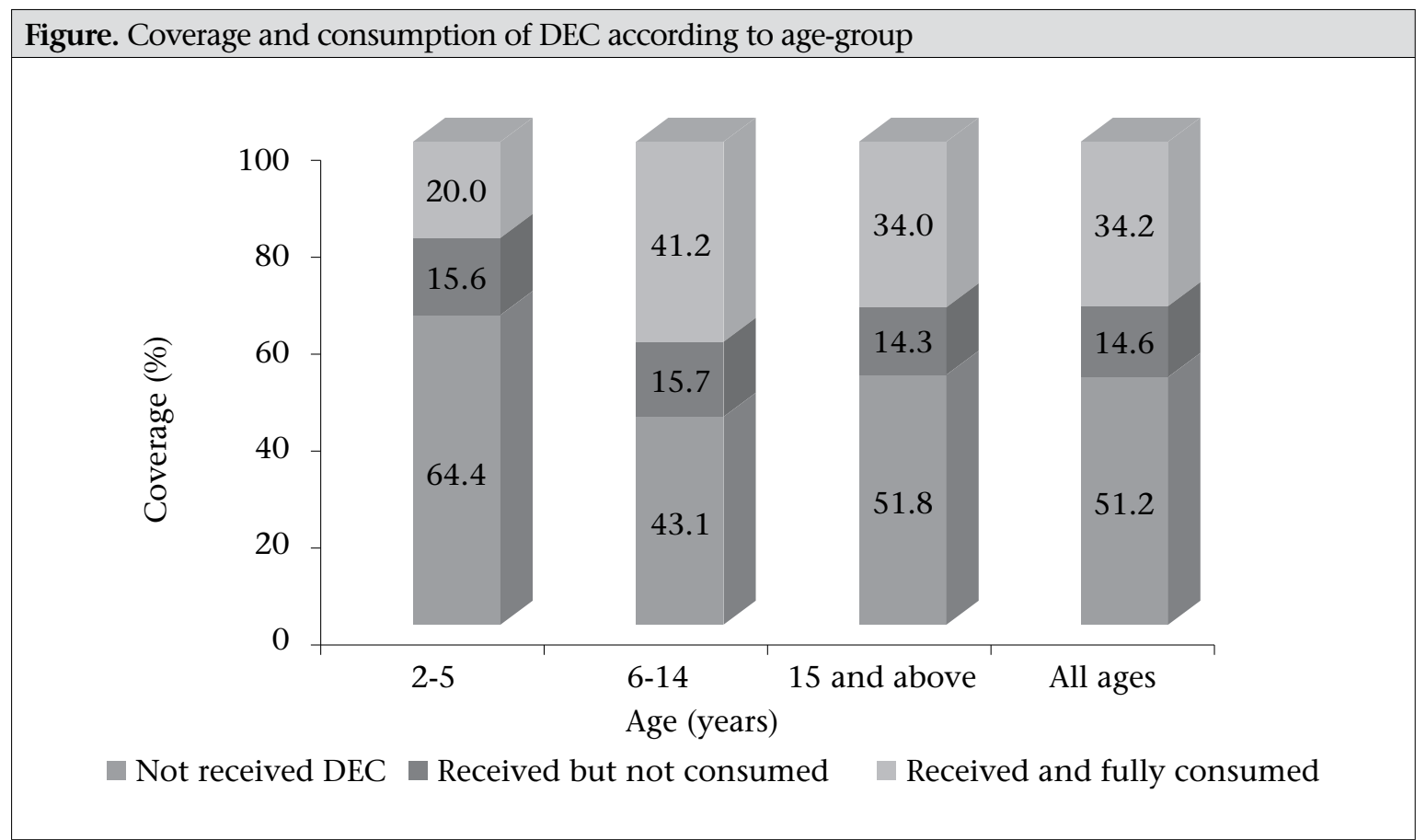

Table 2. Major reasons for non-consumption of $\operatorname{DEC}(n=397)$

\begin{tabular}{|lrc|}
\hline Reason & Number & Percentage \\
\hline No definite reasons & & \\
stated & 103 & 25.94 \\
Fear of side-effects & 80 & 20.15 \\
Do not know MDA & 67 & 16.88 \\
Absent at the time & & \\
of drug distribution/ & & \\
administration & 58 & 14.61 \\
No faith in MDA & 50 & 12.59 \\
Sickness & 14 & 3.53 \\
Others & 25 & 6.30 \\
\hline
\end{tabular}

eligible beneficiaries (25.94\%). Fear of side-effects (20.15\%), lack of awareness of MDA programme (16.88\%), absence at the time of drug distribution (14.61\%), being not convinced about the benefit of MDA (12.59\%) were the common causes of noncompliance. The 'others' category includes some trivial reasons, such as 'forgot to take', 'misplaced the drug', etc.

\section{DEC consumption and side-effects}

We assessed the side-effects after consumption of DEC among the study population. Of 206 persons who consumed DEC, only 6 (2.91\%) reported sideeffects. The most common side-effect was dizziness (4), followed by nausea and/or vomiting (3). These appeared within hours of drug intake and disap- peared within 2-3 days. In none of the cases, any treatment was taken.

\section{Awareness of community regarding MDA}

Data on awareness could be collected from only 131 families. Only the head or any responsible senior member from each family was interviewed to assess the awareness of community regarding filaria and MDA programme (Table 3).

About half of the families (52.67\%) were aware of the existence of filaria as a health problem. Less than one-fifth of the study families (19.85\%) could mention correctly at least one presenting sign or symptom of filaria. Most of the families did not know the role of mosquitoes in the transmission of the disease. Few people explained the association between LF and hydrocele or the role of mosquitoes. About $58 \%$ of the families were aware of MDA programme in the locality. About 58\% respondents knew that drug administration (MDA) was being done in the locality but the majority of them did not know that it was for elimination of LF.

\section{Sources of knowledge}

People were asked about the channel through which they came to know about the MDA programme and which communication method had influenced them. It was observed that majority of respondents (82.89) received information from health personnel through interpersonal channel (Table 4). 


\begin{tabular}{|c|c|c|}
\hline Knowledge & Number & Percentage \\
\hline Heard about filariasis & 69 & 52.67 \\
\hline $\begin{array}{l}\text { Correct knowledge } \\
\text { about at least one } \\
\text { sign or symptom of } \\
\text { filariasis* }\end{array}$ & 26 & 19.85 \\
\hline $\begin{array}{l}\text { Correct knowledge } \\
\text { about transmission of } \\
\text { filariasis (mosquito- } \\
\text { borne) }\end{array}$ & 20 & 15.27 \\
\hline $\begin{array}{l}\text { Correct knowledge } \\
\text { about prevention } \\
\text { (Mosquito control, } \\
\text { MDA) }\end{array}$ & 23 & 17.56 \\
\hline $\begin{array}{l}\text { Heard of MDA pro- } \\
\text { gramme }\end{array}$ & 76 & 58.02 \\
\hline
\end{tabular}

Table 4. Source of information regarding MDA in Burdwan district $(n=76)$

\begin{tabular}{|lcc|}
\hline $\begin{array}{l}\text { Source of } \\
\text { information }\end{array}$ & Number & Percentage \\
\hline $\begin{array}{l}\text { Health personnel } \\
\text { Panchayat }\end{array}$ & 63 & 82.89 \\
representatives & 12 & 15.79 \\
Relatives/neighbours & 9 & 11.84 \\
Mass media & 21 & 27.63 \\
Others & 30 & 39.47 \\
\hline Multiple responses accepted & \\
\hline
\end{tabular}

Some observations on programme implementation

During the coverage survey, we collected additional information on programme implementation in relation to drug distribution component. As per programme guideline, the drug distributors should supervise drug intake to ensure compliance. We conducted in-depth interviews with distributors to collect information on supervised drug intake, timing of drug distribution and the information provided to recipients at the time of drug distribution. Altogether, nine drug distributors worked in the study area; they visited each family only once during daytime. They distributed drugs to individuals present during the visit and handed over the DEC to relatives for those who were absent. Seven out of nine drug distributors advised the beneficiaries to take the drugs after meal. The drug distributors said they could not cover all beneficiaries because of lack of interest among the people and absence at the time of drug distribution.

\section{DISCUSSION}

Although the coverage of drug distribution was $48.76 \%$, the effective coverage was $34.16 \%$ only. Effective coverage rate is the product of coverage by the health system and compliance in the community. A sustainable high coverage of $85 \%$ or more is required for stopping transmission and elimination of disease from the community (4). Other researchers in West Bengal reported more or less similar findings on coverage (9). However, coverage rate reported by other researchers was comparatively higher than the coverage reported in the present study $(10,11)$. The current approach of drug delivery has been found to achieve an effective coverage of $34.16 \%$ only. There is an urgent need for more effective drug-delivery strategies that are adapted to local need.

The proportion of non-compliance varied between $14.25 \%$ and $15.69 \%$ among different age-groups. In the present study, $14.59 \%$ of population failed to consume the drug even after receiving it, which was more or less similar (around 11\%) to that reported in another study (10).

It was stated by drug distributors that they have visited each family only once to distribute the DEC. Repeated contacts may be required to increase the coverage. To cover the absentees and poorlycovered areas, there is provision of 'mop-up' rounds but this activity has not been carried out to maximize the drug consumption. This indicates lack of supervisory activity for the programme. In majority of cases, the DEC intake was not supervised. Similar findings were reported by P. Ray Karmakar et al. (9) and Mahalakshmy T et al. (11).

In the present study, the main reasons for noncoverage were inability of workers to cover entire population, not administering drug to unwilling persons or due to misclassification of persons rendering them not eligible. The important causes of non-compliance were non-supervised drug administration and fear of side-effects. The tablets were distributed during the daytime when most people go out for work, leading to the insufficient coverage. The time for the tablet distribution should be the evening to make it convenient for the community. Seven out of nine drug distributors said 
they thought it was preferable to consume the drugs after meal, and they advised so. This indicates there is need for training before launching the drug distribution programme. A significant proportion of people did not state any definite reason for nonconsumption, indicating lack of motivation.

\section{Side-effects}

Drugs were well-tolerated, and side-effects were negligible. Side-effects reported by other researchers were more or less similar as reported in the present study (9). Although the side-effects were insignificant, fear of side-effects was a major cause of non-compliance. Therefore, it is essential to deliver appropriate health information to address people's concerns and fears about the intervention and make arrangement for the management of cases.

\section{Awareness}

The knowledge gap with regard to the disease and prevailing attitudes and perceptions toward the programme may be a major factor for lower compliance. The awareness about MDA activity was limited among 58\% of families, and among them, the major source of information was health staff. Similar findings have been reported from other studies in India $(9,12)$.

Effective community mobilization activities are essential to strengthen the people's knowledge and to change their perceptions regarding LF. The investigators probed for the participation of the community people in the programme. It was observed that neither the local authorities sought active help or cooperation of the community members nor they had idea about how to involve them in the programme. Lahariya and Mishra also noted similar findings with regard to community participation in their study (13). Involvement of and coordination with other sectors, involvement of NGOs, local leaders, and self-help groups need to be emphasized. An effective health education campaign to make the community aware about LF and increase their participation in the programme is essential to achieve desired success. The focus of the health education should be on locally-appropriate media and announcements by loudspeakers.

It was observed from the study that drug distributors hardly insisted on supervised 'on-the-spot' administration of drugs. Therefore, supervised drug intake was nil or poor in the area. Most respondents who consumed the drug took it after meal; this resulted in poor compliance with drug intake.
The implementation of the programme can be improved by making efficient microplans, ensuring improved supervision, emphasizing the proper training of workers and supervised 'on-the-spot' DEC consumption.

\section{Limitations}

Successful implementation of MDA depends on various preparatory activities, such as selection and availability of health staff and/or volunteers, orientation and training of personnel, mobilization of resources, political commitment, advocacy, and social mobilization. However, in the present study, these components could not be assessed due to resource and time constraints. As several rounds of MDA have already been implemented for the last five years, it is now the time to evaluate the impact of the programme. The prevalence and density of microfilariae, together with drug coverage, are currently the best indicators for measuring the impact of MDA. Research on these issues is needed to explore the current status of the problem and whether or not to continue MDA.

\section{Conclusions}

This evaluation study noted that MDA is restricted to tablet distribution only. The major issues of implementation in compliance, health education, fear of side-effects, motivation/promotion measures, and community participation were not being given due attention. The implementation activities should be strengthened immediately in the MDA programme in India to achieve the goal of LF elimination by 2015 .

\section{REFERENCES}

1. World Health Organization. The regional strategic plan for elimination of lymphatic filariasis: 20102015. New Delhi: Regional Office for South-East Asia, World Health Organization, 2010. 21 p.

2. India. Ministry of Health and Family Welfare. Annual report 2010-2011. New Delhi: Department of Health and Family Welfare, Ministry of Health and Family Welfare, Government of India, 2011. 349 p.

3. India. Ministry of Health and Family Welfare. National Health Policy 2002 (India). New Delhi: Ministry of Health and Family Welfare, Government of India, 2002. 41 p. (http://www.mohfw.nic.in/NRHM/ Documents/National_Health_policy_2002.pdf, accessed on 14 July 2011).

4. India. Ministry of Health and Family Welfare, National Vector Borne Disease Control Programme, Directorate General of Health Services. Guidelines on 
elimination of lymphatic filariasis 2005. New Delhi: National Vector Borne Disease Control Programme, Directorate General of Health Services, Ministry of Health and Family Welfare, Government of India, 2005:7. (http://nvbdcp.gov.in/doc/guidelines-filariasis-elimination-india, accessed on 14 July 2011).

5. Njenga SM, Mwandawiro CS, Wamae CN, Mukoko DA, Omar AA, Shimada M et al. Sustained reduction in prevalence of lymphatic filariasis infection in spite of missed rounds of mass drug administration in an area under mosquito nets for malaria control. Parasit Vectors 2011;4:90.

6. Babu BV, Kar SK. Coverage, compliance and some operational issues of mass drug administration during the programme to eliminate lymphatic filariasis in Orissa, India. Trop Med Int Health 2004;9:702-9.

7. India. Ministry of Health and Family Welfare. Elimination of lymphatic filariasis: training manual on mass drug administration and morbidity management. New Delhi: National Vector Borne Diseases Control Programme, Directorate General of Health services, Ministry of Health and Family Welfare, Government of India, 2004:8-10.

8. World Health Organization. Monitoring and epidemiological assessment of the programme to eliminate lymphatic filariasis at implementation unit level. Geneva: World Health Organization, 2005:1923. (WHO/CDS/CPE/CEE/2005.50).

9. Karmakar PR, Mitra K, Chatterjee A, Jana PK, Bhattacharya S, Lahiri SK. A study on coverage, compliance and awareness about mass drug administration for elimination of lymphatic filariasis in a district of West Bengal, India. J Vector Borne Dis 2011;48:101-4.

10. Kumar P, Prajapati P, Saxena D, Kavishwar AB, Kurian G. An evaluation of coverage and compliance of mass drug administration 2006 for elimination of lymphatic filariasis in endemic areas of Gujarat. Indian J Community Med 2008;33:38-42.

11. Ravish KS, Ranganath TS, Riyaz Basha S. Coverage and compliance of mass drug administration for elimination of lymphatic filariasis in endemic arias of Bijapur district, Karnataka. Int J Basic Med Sci 2011;2:86-89.

12. Rath K, Nath N, Shaloumy M, Swain BK, Suchismita M, Babu BV. Knowledge and perceptions about lymphatic filariasis: a study during the programme to eliminate lymphatic filariasis in an urban community of Orissa, India. Trop Biomed 2006;23:156-62.

13. Lahariya C, Mishra A. Strengthening of mass drug administration implementation is required to eliminate lymphatic filariasis from India: an evaluation study. $J$ Vector Borne Dis 2008;45:313-20. 